\title{
Good Bigrams
}

\author{
Christer Johansson \\ Dept. of Linguistics at Lund University \\ Helgonabacken 12 \\ 22362 Lund, Sweden \\ email: Christer.Johansson@ ling.lu.se
}

\begin{abstract}
A desired property of a measure of connective strength in bigrams is that the measure should be insensitive to corpus size. This paper investigates the stability of three different measures over text genres and expansion of the corpus. The measures are (1) the commonly used mutual information, (2) the difference in mutual information, and (3) raw occurrence. Mutual information is further compared to using knowledge about genres to remove overlap between genres. This last approach considers the difference between two products of the same process (human text-generation) constrained by different genres. The cancellation of overlap seems to provide the most specific word pairs for each genre.
\end{abstract}

\section{Introduction}

Statistical methods have been used to find cohesion between local items of language (such as phonemes, morphemes, or words). Early work (Stolz, 1965; Zellig, 1955) was inspired by the advances in information science (Shannon, 1951; Shannon \& Weaver, 1963). The research benefited from the possibility to store huge amounts of information in computer systems, and the optimism could be overwhelming when the problems were simplified and thought mostly restricted by the size of the corpus. In this paper the stability of some bigram measures will be investigated. Bigrams are items (i.e. word forms) that occur frequently together in a specific order. The meanings of bigrams are not discussed since there is no meaning outside of a context. Co-occurrence is still interesting because bigrams occur non-randomly, sometimes to such an extent that we discern some structure beyond co-occurrence. The reason why it should be so is probably that part of the use of words is reflected by the company that words keep.

Researchers (Church \& Hanks, 1990; Kita $\&$ al., 1994, inter al.) have noted that mutual information tends to be insensitive to high frequency patterns, and unstable for low frequency patterns. Johansson (1994) compared another measure, the difference in mutual information $(\Delta \mu)$, of collocational strength with mutual information $(\mu)$. That measure ranked high frequency bigrams higher than other bigrams if the order was consistent, whereas mutual information tended to pick out combinations of low frequency items. Since low frequency items carry more specific information such bigrams give an illusion of semantic content. It is usually this semantic illusion that we are interested in, but what says that "of the" or "in a" are worse bigrams than "wooden spades" or "various pretexts". Johansson proposed the test of finding some of the characters in the children's story "Alice in Wonderland", and showed that a "new" measure was to some degree "better" than mutual information. Unfortunately, some of that result was based on the fact that mutual information is very sensitive to low frequency items.

\section{Definitions}

\subsection{Mutual information}

In the following $\mathrm{p}(\mathrm{x})$ will denote the observed probability as defined by $\mathrm{p}(\mathrm{x})=\mathrm{F}(\mathrm{x}) / \mathrm{N}$ where $F(x)$ is the frequency of occurrence of $x$, and $\mathrm{N}$ is the number of observed cases. $\mathrm{N}$ is, in the calculations, equal to the corpus size in words. Given this, the mutual information ratio (Church \& Hanks, 1990; Church \& Mercer, 1993; Steier \& Belew, 1991) is expressed by Formula 1. (Church \& Hanks refer to this measure as the association ratio for technical reasons). 


$$
\begin{aligned}
& \mu=\log _{2}\left(\frac{p\left(\left[w_{1}, w_{2}\right]\right)}{p\left(w_{1}\right) p\left(w_{2}\right)}\right) \approx \\
& \approx \log _{2}\left(\frac{N^{*} O c c\left(\left[w_{1}, w_{2}\right]\right)}{O c c\left(w_{1}\right) O c c\left(w_{2}\right)}\right)
\end{aligned}
$$

Formula 1: The mutual information ratio

The instability of statistical measures seems to be a problem in statistical bigrams. Especially low frequency counts cause instability. To avoid this use the rule of thumb that a bigram must occur more than four times (cf. Church \& Hanks, 1990:p.24) to be considered as a candidate for an interesting bigram.

\subsection{The difference in mutual informa- tion: temporal co-occurrence}

A reasonable way of using the temporal ordering in word pairs is to consider the opposite ordering of the word pair as negative evidence against the present order. $\Lambda$ reasonable measure would be to use the difference in mutual information between the two orderings, hereafter $\Delta \mu$. The size of the corpus cancels out and $\Delta \mu$ can be calculated by a ratio between frequencies. This is intuitively correct for a comparison between apples and pears, i.e. you can say that apples (w1 w2) occur twice as often as pears (w2 w1) in my fruit bowl (corpus). ( $\mathrm{p}$ is the probability in the fixed corpus $(\mathrm{f} / \mathrm{N})$ which is different from the probability in the language. It is impossible to have a fixed corpus that equals the language since language does not have a fixed number of words or word patterns).

\subsubsection{Handling zero negative evidence}

In the case that the reversed ordering of a word pair has not been observed in the corpus, the measure becomes undefined. To relieve this the frequency ${ }^{1}$ is multiplied by a constant (10), and the frequency of the reversed ordering is set to 1 . Subtracting 9 from that value does not add anything to the measure for a single occurrence $(\log (10-9)=0)$.

Other ways of handling zero-frequencies are evaluated in (Gale \& Church, 1994), e.g. the Good-Turing method. Relative frequencies of non-observed word pairs are hard to estimate. For example, the frequencies of frequencies (X) and frequency ( $\mathrm{Y}$ ) used in the

\footnotetext{
${ }^{1}$ I will use 'frequency' as equivalent to 'occurrence' in the sample corpus.
}

Good-Turing method are linearly dependent in a log-log scale, i.e., there is an infinite frequency of non-observed items (which is another way of saying that we cannot expect the unexpected).

$$
\Delta \mu=\left\{\begin{array}{c}
\log _{2}\left(\frac{\operatorname{Occ}\left(\left[w_{1}, w_{2}\right]\right)}{\operatorname{Occ}\left(\left[w_{2}, w_{1}\right]\right)}\right) \\
: \text { if } \operatorname{Occ}\left(\left[w_{2}, w_{1}\right]\right)>0 \\
\log _{2}\left(10 * O c c\left(\left[w_{1}, w_{2}\right]\right)-9\right) \\
\text { if } \quad \operatorname{Occ}\left(\left[w_{2}, w_{1}\right]\right)=0
\end{array}\right.
$$

Formula 2: Handling zero frequencies

\section{Illustration}

The difference between the two measures are perhaps best illustrated with some concrete examples. In a previous paper (Johansson, 1994) "Alice's adventures in Wonderland" (AIW) was used as an experimental corpus to compare phrase finding for $\mu$, and a new measure $-\Delta \mu$. A critique against that corpus is that the corpus is very small. "Through the Looking Glass" and "The Hunting of the Snark" extend that corpus to about 63000 words of which 26831 occurred more than 4 times. With the criterion that an interesting bi-

\begin{tabular}{|c|c|c|}
\hline $\begin{array}{c}\text { Effect of } \\
\text { delta }\end{array}$ & $\begin{array}{c}\text { Effect of } \\
\text { mu }\end{array}$ & bigram \\
\hline 215 & 1883 & cheshire cat \\
\hline 7 & 34 & humpty dumpty \\
\hline 48 & 202 & looking glass \\
\hline 33 & 136 & march hare \\
\hline 7 & 28 & mock turtle \\
\hline 204 & 1400 & red king \\
\hline 7 & -9 & red queen \\
\hline 29 & -519 & the dormouse \\
\hline 174 & 931 & white king \\
\hline 160 & 932 & white knight \\
\hline 29 & 5 & white queen \\
\hline 47 & 190 & white rabbit \\
\hline
\end{tabular}
gram occurs more than 4 times 1970 bigram candidates were found in this larger corpus.

In the previous table the effect is measured by the number of steps a bigram is moved up compared to a sorted frequency list. The effect of mutual information under these conditions is higher than the proposed measure for finding most characters in AIW, except for some names defined by definite article + noun, and common adjective + noun. 


\section{Material}

In the rest of this paper, the corpus is the SUSANNE corpus (Sampson, 1994). This corpus consists of an extensively tagged and annotated subset from the Brown Corpus of American English. The corpus is fairly small, but provides information on grammatical roles on the word and phrase level. This makes the SUSANNE corpus suitable for further research.

The SUSANNE corpus is divided into 4 (approximately equally large) genre subcategories:

"A: press reportage

G: belles lettres, biography, memoirs

$\mathrm{J}$ : learned (mainly scientific and

technical) writing

$\mathrm{N}$ : adventure and Western fiction"

(Sampson, 1994:p.174)

Each genre has approximately 20,000 unique word pairs ${ }^{2}$. The four genres will be used as one factor in the comparison between different measures. The question is whether the genre interacts with the ability of the different measures to discover bigrams. In category $\mathrm{A}$ 439 unique bigrams (occurring more than 4 times) were found, in $\mathrm{G} 486$, in $\mathrm{J} 598, \mathrm{~N}$ 620 , and 2573 for the used corpus ${ }^{3}$.

\section{Method}

The highest ranking bigrams according to the measure are sampled at 5 different levels: the $10,50,100,200$ and 400 top collocations. Samples are sorted and compared for overlap by the UNIX command 'comm -12 SAMPLE1 SAMPLE2 | wc -l', and the percentage of overlap was calculated from the size of the sample.

Stability of bigrams was tested by three different overlaps. 1) The overlap between samples from genres, and samples for the entire corpus for the same measure. 2) The overlap between different measures at the five different levels for the different genres and the entire corpus. 3) The overlap between different genres.

${ }^{2}$ (A 21198 unique / 29969 total / 5332 unique words; G 22248 / 31006 / 6048; J 19039 / 29484/4676; N 20902 / 31959 / 4876; all 74126 / 122421 / 13458)

${ }^{3}$ The last small part of each genre was excluded from the start for future purposes.

\section{Results}

\subsection{Mutual Information}

The average overlap between genres and the corpus showed that the $\mathbf{J}$ sample was much more stabile than the other genres ${ }^{4}$. The $J$ genre would be the genre that information retrieval applications would be most interested in. The ranking of the genres according to the stability of the overlap is: JANG. The highest collocations are most stabile for J, where the other genres show less specificity (i.e. equal or growing percentages as the overlap grows).

\begin{tabular}{|c|c|c|c|c|c|c|}
\hline 10 & 50 & 100 & 200 & 400 & mean & \\
\hline 20 & $\overline{22}$ & 30 & 27.5 & 21.5 & $\overline{24.2}$ & A \\
\hline 0 & 6 & 10 & 14.5 & 16.7 & 9.4 & $\bar{G}$ \\
\hline 60 & 62 & 48 & 36.5 & 31 & 47.5 & $\mathrm{~J}$ \\
\hline 10 & 6 & 7 & 15 & 22 & 12.0 & $\mathrm{~N}$ \\
\hline
\end{tabular}

\subsection{Delta Mutual Information}

Delta mutual information shows little effect of genre, and sample size. Growing sample size predicts less overlap. The ranking of genres is: GANJ. Delta mutual information seems to rank the less specific genres high.

\begin{tabular}{|c|c|c|c|c|c|c|}
\hline$\overline{10}$ & 50 & 100 & 200 & 400 & mean & \\
\hline$\overline{70}$ & 64 & $\overline{53}$ & 47.5 & 444.2 & 55.7 & $\mathrm{~A}$ \\
\hline 60 & 58 & 54 & 58.5 & 51.5 & 56.4 & $\mathrm{G}$ \\
\hline 60 & 54 & 48 & 43 & 39.2 & 48.8 & $\mathbf{J}$ \\
\hline 50 & 52 & 49 & 51 & 45.5 & 49.5 & $\mathrm{~N}$ \\
\hline
\end{tabular}

A factorial ANOVA on measure and genre shows that there is a significant effect $(p<0.001)$ of measure $(\Lambda \mu$ or $\mu)$, genre and interaction between measures. $\mathrm{F}$ (measure, $1 \mathrm{df})=136.2, \mathrm{~F}$ (genre, 3df $)=9.8, \mathrm{~F}$ (measure, genre, 1,3$)=15.4, p<0.001$. These two measures are significantly different.

\subsection{Occurrence}

The results for the samples are similar to $\Delta \mu$. The overlap is generally higher for occurrence than $\Delta \mu$, but the ranking of genres is the same: GANJ. An ANOVA on measure $(\Delta \mu$ and occurrence) and genre show less significant effect on measure, and no significant effect of genre, or interaction (these measures behave in the same direction).

\footnotetext{
4In preliminary investigations the $\mathrm{J}$ genre was the least stabile genre for mutual information. This was 'corrected' by the demand that candidate bigrams should occur more than 4 times.
} 


\begin{tabular}{|l|l|l|l|l|l||c|}
\hline 10 & 50 & 100 & 200 & 400 & mean & \\
\hline \hline 60 & 70 & 65 & 60.5 & 51 & 61.3 & $\mathrm{~A}$ \\
\hline 60 & 70 & 69 & 65.5 & 61 & 65.1 & $\mathrm{G}$ \\
\hline 70 & 62 & 53 & 48.5 & 43.5 & 55.4 & $\mathrm{~J}$ \\
\hline 70 & 64 & 57 & 54.5 & 54.2 & 59.9 & $\mathrm{~N}$ \\
\hline
\end{tabular}

$\mathrm{F}($ measure, $1 \mathrm{df})=11.1 \mathrm{p}<0.02, \mathrm{~F}$ (genre, $3 \mathrm{df})=2.7, \mathrm{p}>0.05, \mathrm{~F}$ (measure, genre, 1,3 ) $=0.218, p>0.8$. Occurrence is significantly more stabile than the other measure, but there is only a small difference of genres (occurrence and $\Delta \mu$ react in a similar way to genre - i.e. on high occurrence).

\subsection{Comparison between measures}

The overlap between measures is calculated for all combinations of measures. At the higher levels a high overlap can be expected since there is little possibility to fall out (e.g. in A 400 out of 439 is $91 \%$ of the sample). The results from this test indicate that the overlap between $D(\Delta \mu)$ and $F$ (occurrence) is significantly and consistently higher than between the other combinations (especially for the entire corpus).

\begin{tabular}{|c|c|c|c|c|c|c|c|}
\hline 10 & 50 & 100 & 200 & 400 & Genre & Test & $\begin{array}{r}\text { mean } \\
\text { over- } \\
\text { lap }\end{array}$ \\
\hline 0 & 6 & 22 & 44.5 & 93.2 & $\mathrm{~A}(439)$ & $\mathrm{M}=\mathrm{D}$ & 33.1 \\
\hline 0 & 6 & 16 & 37.5 & 91.0 & A & $\mathrm{M}=\mathrm{F}$ & 30.1 \\
\hline 90 & 64 & 74 & 78.0 & 91.2 & A & $D=F$ & 79.4 \\
\hline 0 & 18 & 23 & 45.5 & 86.0 & $\mathrm{G}(486)$ & $\mathrm{M}=\mathrm{D}$ & 34.5 \\
\hline 0 & 14 & 20 & 43.0 & 82.0 & $\mathrm{G}$ & $\mathrm{M}=\mathrm{F}$ & 31.8 \\
\hline 80 & 76 & 78 & 77.5 & 84.0 & $G$ & $D=F$ & 79.1 \\
\hline 0 & 8 & 13 & 34.0 & 72.2 & $\mathrm{~J}(598)$ & $\mathrm{M}=\mathrm{D}$ & 25.4 \\
\hline 0 & 4 & 11 & 28.5 & 64.0 & $\mathrm{~J}$ & $\mathrm{M}=\mathrm{F}$ & 21.5 \\
\hline 60 & 84 & 78 & 72.5 & 75.5 & $J$ & $D=F$ & 74.0 \\
\hline 0 & 8 & 22 & 33.5 & 70.5 & $\mathrm{~N}(620)$ & $\mathrm{M}=\mathrm{D}$ & 26.8 \\
\hline 0 & 6 & 20 & 28.0 & 63.7 & $\mathrm{~N}$ & $\mathrm{M}=\mathrm{F}$ & 23.5 \\
\hline 40 & 68 & 71 & 67.0 & 72.5 & $N$ & $D=F$ & 63.7 \\
\hline 0 & 0 & 1 & 7.0 & 15.7 & $\operatorname{all}(2573)$ & $\mathrm{M}=\mathrm{D}$ & 4.7 \\
\hline 0 & 0 & 1 & 4.0 & 13.0 & all & $\mathrm{M}=\mathrm{F}$ & 3.6 \\
\hline 40 & 54 & 58 & 58.0 & 59.5 & all & $D=F$ & 53.9 \\
\hline
\end{tabular}

\subsection{Overlap between genres}

To estimate the overlap of the genres the number of common bigrams between two genres were found and compared to the size of the smallest genre. The results indicate an average overlap between the genres of $10 \%$.

\begin{tabular}{|l|l|l|l|l|}
\hline \multicolumn{5}{|c|}{ Overlap of genres (\% of smallest genre) } \\
\hline & A & G & J & N \\
\hline A & - & & & \\
\hline G & 11.0 & - & & \\
\hline J & 9.4 & 11.0 & - & \\
\hline N & 10.0 & 12.0 & 7.5 & - \\
\hline
\end{tabular}

\subsection{Reduction of the bigrams}

The bigrams that are rated high by the measures (especially mutual information) are mixed between two different types of bigrams: (1) bigrams with high internal cohesion between low frequency items that may be associated with a specific interpretation (e.g. "carbon tetrachloride" or "cheshire cat"), (2) bigrams with high internal cohesion with usually high frequency of both items that may be associated with a "syntactical" interpretation (e.g. "in the").

To separate type 1 from type 2 some information about the overlap of genres might be used. The type 2 bigrams are typically found in most genres, whereas type 1 bigrams are specific to a text. The results above indicate that we can use the genres with least overlap to filter out common bigrams (i.e. A use $J, G$ use J, J use N, N use J).

In the following table the effect of the genre (column 2) is shown by the number of 'surviving' bigrams from the candidate bigrams (column 1). The third column shows the effect of removing the bigrams that occur (more than 4 times) in both directions after common bigrams have been removed (first parenthesis shows actual removed, second shows those that would have been removed (i.e. those bigrams with both orderings in the candidate set). The fourth column shows the effect of removing bigrams that contains words that occur more than 4 times in the rest of the corpus (i.e. in A $\mathrm{G} \mathrm{N}$ for J) after the bigrams have been formed. The reason for filtering after forming bigrams is that words that are filtered out later work as place holders, and prevent some bigrams to form. 'The reduction is most notable for removing bigrams that contain common words between genres: genre $\mathrm{G}$ and $\mathrm{N}$ contain few good candidates of collocations type 1 .

\begin{tabular}{|c|c|c|c|c|}
\hline Cand. & Genre & Word order filter & $\begin{array}{c}\text { Freq. } \\
\text { words }\end{array}$ & \\
\hline$\overline{439}$ & 216 & $179(-63)(-80)$ & 12 & $\mathrm{~A}$ \\
\hline 486 & 159 & $119(-40)(-127)$ & 1 & $\mathrm{G}$ \\
\hline 598 & 355 & $277(-78)(-131)$ & 37 & $J$ \\
\hline 620 & 395 & $291(-104)(-159)$ & 0 & $\mathrm{~N}$ \\
\hline
\end{tabular}


The following bigrams survived the harshest condition of removing bigrams containing words of other genres. (Genre J, later ordered by mutual information). Some good candidates were (of course) removed, e.g. "black body", "per cent", "united states".

$\begin{array}{ll}\text { 12.2 poynting robertson } & 9.1 \text { pulmonary vein } \\ \text { 11.8 indirect coombs } & 8.9 \text { active agent } \\ \text { 11.6 burning arcs } & 8.9 \text { bronchial artery } \\ 11.4 \text { anionic binding } & 8.9 \text { liquid phase } \\ \text { 11.1 binding capacity } & 8.8 \text { pulmonary artery } \\ 11.0 \text { starting buffer } & 8.6 \text { anode holder } \\ \text { 10.7 antenna beam } & 8.3 \text { solar radiation } \\ \text { 10.6 wave lengths } & 8.2 \text { reaction tubes } \\ \text { 10.3 wave length } & 8.0 \text { quadric surface } \\ \text { 10.1 multiple secant } & 7.8 \text { brightness tempera- } \\ & \text { ture } \\ \text { 10.0 carbon tetrachloride } & 7.8 \text { mass flow } \\ 9.9 \text { bronchial arteries } & 7.7 \text { gas phase } \\ 9.9 \text { heat transfer } & 7.7 \text { surface cleaning } \\ 9.9 \text { ideal gas } & 7.1 \text { reaction cells } \\ 9.8 \text { agglutinin activity } & 7.1 \text { surface active } \\ 9.5 \text { hydrogen atoms } & 6.7 \text { artery pulmonary } \\ 9.4 \text { multiple secants } & 5.0 \text { anode surface } \\ 9.3 \text { antibody activity } & 4.7 \text { surface temperature } \\ \text { 9.1 particle size } & \end{array}$

In the A genre (News) the following 12 bigrams survived:

\begin{tabular}{|ll} 
12.5 anne arundel & 8.9 sales tax \\
12.0 rhode island & 8.9 payroll tax \\
10.0 grand jury & 8.2 fulton county \\
9.9 rule charter & 8.0 football league \\
9.2 austin texas & 7.5 kennedy administra- \\
& tion \\
8.9 sunday sales & 7.3 tax bill
\end{tabular}

Genres $\mathrm{G}$ and $\mathrm{N}$ contain few candidates for collocations (among the 'best' ones in $\mathrm{N}$ were "gray eyes", "picked up", "help me" and "stared at" which are quite telling about the prototypical western story: "The gray eyes stared at the villain who picked up his knife, while the girl cried "help me"."

\section{Other approaches}

The temporal dependencies of an ordered collocation [word1, word2] has been seen as a problem since the theory of mutual information assumes the frequencies of word pairs to be symmetric (i.e., $f([\mathrm{w} 1, \mathrm{w} 2])$ and $\mathrm{f}([\mathrm{w} 2$, w1]) to be equal). Delta mutual information relies on this difference in temporal ordering.
"[ $[..] \mathrm{f}(\mathrm{x}, \mathrm{y})$ encodes linear precedence. [...] Although we could fix this problem by redefining $f(x, y)$ to be symmetric (by averaging the matrix with its transpose), we have decided not to do so, since order information appears to be very interesting." (Church \& Hanks, 1990:p.24)

Merkel, Nilsson, \& Ahrenberg (1994) have constructed a system that uses frequency of recurrent segments to determine long phrases. In their approach they have to chunk the text into contiguous segments. Significant frequency counts are achieved through the use of a very large corpus, and/or a corpus specialised for a specific task. They report that it was possible for them to divide a large corpus into smaller sub-sections with little loss.

Smadja (1993) finds significant bigrams using an estimate of $\mathrm{z}$-score (deviation from an expected mean). Smadja's method seems to require very large corpora, since the method needs to estimate a reliable measure of the variance of the frequencies with which words co-occur. This makes the method dependent on the corpus size. Smadja reports the use of a corpus of size 10 million words.
"More precisely, the statistical methods we use do not seem to be effective on low frequency words (fewer than 100 occurrences)." (Smadja, 1993:p.168)

Kita \& al. (1994) proposed another measure of collocational strength that was based on the notion of a reduction in 'processing cost' if a frequent chunk of text can be processed as one chunk. Cost reduction tended to extract conventional 'predicate phrase patterns', e.g., "is that so" and "thank you very much".

Steier \& Belew (1991) discuss the 'exporting' of phrases into a general vocabulary, where a word pair with high mutual information within a topic tends to have lower mutual information within the collection, and vice versa. They relate a higher mutual information within a topic than in the collection to a lower value of discrimination.

Church \& Gale (1995) have found it useful to compare the distribution of terms across documents. They showed that a distribution different from what could be expected by a (random) Poisson process indicates interesting terms. This approach is similar to the use of one genre to find interesting items in 
another. However, removal of the overlap needs some knowledge about the genres apart from checking explicitly for a genre with least overlap. Cancelling overlap has the advantage that it can cancel out similar underlying causes, while it exaggerates the underlying causes that differ between genres. Some questions remain: at which level should overlap be formed? overlap in words or in bigrams; how many repetitions does it take for a word or bigram to 'belong' to a genre?

\section{Conclusion}

The question is "what is gained by using a measure?". Mutual information tends to find combinations of words that are highly co-ordinated with each other, but these bigrams show both interesting bigrams (c.g. "cheshire cat") and conventional (and uninteresting for keywords) bigrams (e.g. "in a"). The stability of interesting bigrams is improved by demanding candidate bigrams to occur more than a fixed number of times.

In this paper it has been shown that genre matters, and can be used to extract items that differ between genres. Instead of balancing one big corpus, the analysis of one corpus might benefit from finding out how it is different from another corpus. The bigrams that were formed by using different genres as filters showed interesting characteristics.

However, if we are to deal with larger amounts of data it might be unrealistic to compare differences directly between two large genres without the exclusion of terms that occur by chance.

The method that could be recommended from the results presented in this study is to triangulate a sample by the difference to other genres that we have some meta-knowledge about (i.e. we know that Western Fiction and Scientific Writing, at least on the surface, have little vocabulary in common).

\section{References}

Church, K., \& Gale, W. (1995). Inverse Document Frequency (IDF): A Measure of Deviations from Poisson. D. Yarowsky \& K. Church (Eds.), Third Workshop on Very Large Corpora (pp. 121-130), MIT, Cambridge, Mass.

Church, K., \& Hanks, P. (1990). Word Association Norms, Mutual Information, and Lexicography. Computational Linguistics, 16(1), 22-29.

Church, K., \& Mercer, R. (1993). Introduction to the special issue on Computational Linguistics using Large Corpora. Computational Linguistics, 19(1), 1-24.

Gale, W., \& Church, K. (1994). What is wrong with adding one? In N. Oostdijk \& P. de Haan (Eds.), Corpus based research into language (pp. 189-198). Amsterdam - Atlanta: Rodopi.

Johansson, C. (1994). Catching the Cheshire Cat, Coling (pp. 1021-1025), Kyoto, Japan.

Kita, K., Omoto, T., Yano, Y., \& Kato, Y. (1994). Application of Corpora in Second Language Leatning - The Problem of Collocational Knowledge Acquisition --, se cond annual workshop on very large corpora (pp. 43-56), Kyoto, Japan.

Merkel, M., Nilsson, B., \& Ahrenberg, L. (1994). A phrase-retricval system based on recurrence, second annual workshop on very large corpora (pp. 99-108), Kyoto, Japan.

Sampson, G. (1994). SUSANNE: A Domesday Book of English Grammar. In N. Oostdijk \& P. de Haan (Eds.), Corpus-based research into language (pp. 169-187). Amsterdam - Atlanta.

Shamnon, C.E. (1951). Prediction and Entropy of printed English. Bell Systems Technical Journal, 30(1), 50-65.

Shannon, C.E., \& Weaver, W. (1963). The Mathematical Theory of Communication. Urbana: University of Illinois Press.

Smadja, F. (1993). Retrieving Collocations from Text: Xtract. Computational Linguistics, $19(1), 143-177$.

Steier, A.M., \& Belew, R.K. (1991). Exporting phrases: A statistical analysis of topical language. R. Casey \& B. Croft (Eds.), 2 nd Symposium on Document Analysis and Information Retrieval.

Stolz, W. (1965). A probabilistic procedure for grouping words into phrases. Language and Speech, 8, 219-235.

Zellig, H. (1955). From phoneme to morpheme. Language, 31, 190-222. 